\title{
Gemeinsam für die Onkologie
}

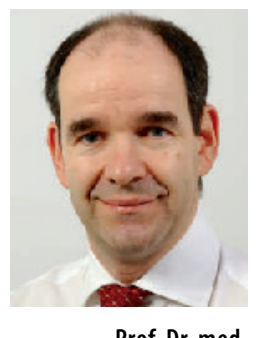

Prof. Dr. med. Frank Zimmermann Chefarzt der Abt. Radioonkologie Universitätsspital Basel

E-Mail: f.zimmermann @uhbs.ch
$\mathrm{M}$ ehr als 700 Referenten der medizinischen Onkologie und der Hämatologie, vertreten in den deutschen, österreichischen und schweizerischen Gesellschaften für Hämatologie und Onkologie, treffen sich Anfang Oktober in Basel für ihre Jahrestagung.

Den Organisatoren ist es gelungen, in den wenigen, zur Verfügung stehenden Tagen ein umfängliches Programm für die 4.500 erwarteten Teilnehmer des Kongresses zu erstellen.

Neben den großen, immer noch weltweit führenden onkologischen Erkrankungen wie dem Lungen-, Mamma- oder Prostatakarzinom bietet sich ausreichend Raum für die Darstellungen der Innovationen der letzten Jahre, die aktuellen Problemstellungen aber auch die möglichen zukünftigen diagnostischen und therapeutischen Wege.

Es gibt genügend Platz, um Tumorerkrankungen von den Anfängen des Screenings bis zur detaillierten Nachsorge, von der Kuration bis zu Palliation, von der handfesten onkologischen Therapie bis hin zu begleitenden supportiven Maßnahmen darzustellen. Zudem wird den bedeutsamen Aspekten der geriatrischen Onkologie und der psychoonkologische Führung von Patienten sowie der vorurteilsfreie Interpretation von Studiendaten und deren Relevanz für die tägliche klinische Routine ein Forum geboten.

Es ist ein guter Wurf gelungen in einer Mischung von wissenschaftlichem Symposium, Fortbildungen und freien Vorträgen, ohne die Sponsoren des Kongresses mit ihren Belangen zur vernachlässigen.
Problematisch erscheint bei diesem umfänglichen Programm einzig, ob es den Teilnehmern an Ausdauer und an Gelegenheit zur vertiefenden Diskussion reichen wird, und die gebotenen Chancen der Vielfalt somit wirklich zufriedenstellend genutzt werden können.

Wenn zum Abschluss der Veranstaltung so renommierte Referenten wie die Herren Cavali und Tannock ihre Gedanken über die zukünftige Entwicklung in der medizinischen Onkologie und die Fortschritte in der Krebstherapie abwägend und kritisch darlegen, darf man vor allem auf eine kritische Reflexion hoffen.

Antworten und Lösungen auf Fragen wie - „Welche der modernen Therapien können wir uns leisten, im Zeitalter einer zunehmenden Alterung der Gesellschaft, der täglichen Diskussionen um die Wirtschaftslage selbst in den wohlhabenden westlichen Ländern, und die Forderung des mündigen, aber nicht immer objektiv informierten Patienten nach immer mehr, immer früherer und immer intensiverer Betreuung “ - dürfen wir an diesem Tag zweifellos nicht erwarten, aber zumindest eine fokussierte Auseinandersetzung mit dem wohl brennendsten Problem in der Onkologie.
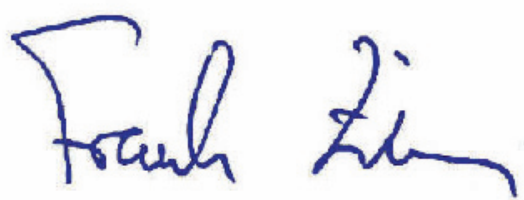